\title{
Magnetic-field-induced spontaneous superlattice formation via spinodal decomposition in epitaxial strontium titanate thin films
}

\author{
Naoki Wakiya ${ }^{1,2,3}$, Naonori Sakamoto ${ }^{1,3}$, Shota Koda ${ }^{3}$, Wataru Kumasaka ${ }^{3}$, Nipa Debnath ${ }^{2}$, \\ Takahiko Kawaguchi ${ }^{3}$, Takanori Kiguchi ${ }^{4}$, Kazuo Shinozaki ${ }^{5}$ and Hisao Suzuki ${ }^{1,2,3}$
}

Periodically structured nanomaterials such as superlattices have a wide range of applications. Many electronic devices have been fabricated from these materials. The formation of spontaneous layer structures using epitaxial growth has also been reported for many compound semiconductors but for very few ceramics. We demonstrate that strontium titanate (Sr-Ti-O) thin films having an A-site excess composition in the perovskite structure deposited by pulsed laser deposition under a magnetic field show a spontaneously formed superlattice structure. The spontaneous superlattice formation mechanism has been proven to exhibit spinodal decomposition. Preparation of a part of the phase diagram for Sr-Ti-O thin films is reported for the first time. Although $\mathrm{SrTiO}_{3}$ bulk is quantum paraelectric, previous reports have described that strained $\mathrm{SrTiO}_{3}$ thin films show room-temperature ferroelectricity, especially along the in-plane direction. However, induced ferroelectricity along the out-of-plane direction has been reported in films with a limited thickness of less than $10 \mathrm{ml}$. The results show that the Sr-Ti-O thin films with spontaneously formed superlattice structures exhibit room-temperature ferroelectricity even when $300 \mathrm{~nm}$ thick. The induced ferroelectricity is brought about by the strain along the out-of-plane direction and is explained based on thermodynamic considerations.

NPG Asia Materials (2016) 8, e279; doi:10.1038/am.2016.76; published online 17 June 2016

\section{INTRODUCTION}

Spontaneous superlattice formation during thin film deposition by molecular beam epitaxy or metal-organic vapor phase epitaxy is known to occur in semiconductors, such as $\mathrm{AlGaAs},{ }^{1} \mathrm{AlGaN},{ }^{2}$ InGaN, ${ }^{3} \mathrm{ZnSeTe}^{4}$ and SiGe. ${ }^{5}$ In these films, periodic compositional modulations have been reported along the vertical direction of the substrates. Therefore, a semiconductor thin film with $A_{x} B_{1-x}$ composition undergoes phase separation into a stacked structure with A-excess and B-excess layers. It is extremely interesting that the spontaneously formed periodical structure affects the band gap, transport properties and optical properties of the semiconductors. ${ }^{6,7}$ Two possible mechanisms of such spontaneously formed periodical structures are proposed. One proposed mechanism is nucleation and growth. Another proposed mechanism is spinodal decomposition. Daruka and Tersoff $^{8}$ theoretically examined the dynamics of thin film growth by vapor deposition and bulk diffusion. They predicted that spontaneous superlattice formation can occur by spinodal decomposition if the deposition rate is intermediate. Spontaneous superlattice formation is also reported for metal alloy $(\mathrm{Au}-\mathrm{Ni})$ and carbide (TiC) thin films deposited under an ion-impingement (bombardment) atmosphere. ${ }^{9,10}$ In their works, additional kinetic energy by ion-impingement is regarded as having a lower activation energy of up-hill diffusion, which is characteristic of spinodal decomposition. For oxides, few reports describe spontaneous superlattice formation during thin film deposition. One example is a $\left(\mathrm{HfO}_{2}\right)_{0.25}\left(\mathrm{SiO}_{2}\right)_{0.75}$ thin film produced by atomic layer deposition. ${ }^{11}$ Because $\left(\mathrm{HfO}_{2}\right)_{0.25}\left(\mathrm{SiO}_{2}\right)_{0.75}$ forms a solid solution, the spontaneous superlattice formation mechanism is regarded as spinodal decomposition. Another example is the $\left(\mathrm{YBa}_{2}-\right.$ $\left.\mathrm{Cu}_{3} \mathrm{O}_{7-\mathrm{x}}\right)_{0.5}\left(\mathrm{BaZrO}_{3}\right)_{0.5}$ thin film produced by PLD. ${ }^{12}$ This film comprises alternating layers of $\mathrm{YBa}_{2} \mathrm{Cu}_{3} \mathrm{O}_{7-\mathrm{x}}$ and $\mathrm{BaZrO}_{3}$. Because $\mathrm{YBa}_{2} \mathrm{Cu}_{3} \mathrm{O}_{7-\mathrm{x}}$ and $\mathrm{BaZrO}_{3}$ do not form a solid solution, the spontaneous superlattice formation mechanism is believed to be pseudospinodal decomposition by which both spinodal decomposition and nucleation and growth occur simultaneously. ${ }^{13}$ Another example is the $\mathrm{Ba}-\mathrm{Sn}-\mathrm{O}$ thin film with long-range spontaneous ordering produced by PLD. ${ }^{14}$ The Ba-Sn-O thin films have a Ba-excess composition. The superlattice origin was ascribed to the Ruddlesden-Popper (RP) phase, ${ }^{15}$ which is well known in Sr-Ti-O systems with the general formula of $\mathrm{Sr}_{1+\mathrm{n}} \mathrm{Ti}_{\mathrm{n}} \mathrm{O}_{3 \mathrm{n}+1}$ or $\left(\mathrm{SrTiO}_{3}\right)_{\mathrm{n}} \bullet(\mathrm{SrO})(n=1,2,3, \ldots \infty)$. These phases consist of an additional SrO layer inserted into the $\mathrm{SrTiO}_{3}$ lattice, thereby forming a SrO bilayer. Among these phases, $n=\infty$ denotes $\mathrm{SrTiO}_{3}$. Although pure $\mathrm{SrTiO}_{3}$ is quantum paraelectric, ${ }^{16}$ ferroelectricity is induced by an electric field, ${ }^{17} \mathrm{Ca}$ substitution $^{18}$ and oxygen isotope exchange. ${ }^{19}$ Stress-induced

${ }^{1}$ Research Institute of Electronics, Shizuoka University, Hamamatsu, Japan; ${ }^{2}$ Graduate School of Science and Technology, Shizuoka University, Hamamatsu, Japan; ${ }^{3}$ Department of Electronics and Materials Science, Shizuoka University, Hamamatsu, Japan; ${ }^{4}$ Institute for Materials Research, Tohoku University, Sendai, Japan and ${ }^{5}$ School of Materials and Chemical Technology, Tokyo Institute of Technology, Tokyo, Japan

Correspondence: Professor N Wakiya, Research Institute of Electronics, Shizuoka University, 3-5-1 Johoku Naka-ku, Hamamatsu 432-8561, Japan.

E-mail: wakiya.naoki@shizuoka.ac.jp

Received 8 July 2015; revised 24 March 2016; accepted 14 April 2016 
ferroelectricity in $\mathrm{SrTiO}_{3}$ was reported first by Uwe and Sakudo, ${ }^{20}$ based on Landau-Devonshire-type phenomenological theory (thermodynamic calculation). Haeni et al. ${ }^{21}$ extended this calculation to predict room-temperature ferroelectricity in strained $\mathrm{SrTiO}_{3}$. On the basis of their prediction, they deposited a $\mathrm{SrTiO}_{3}$ thin film with in-plane strain (tensile strain) onto a $\mathrm{DyScO}_{3}$ (110) substrate and observed in-plane ferroelectricity. This effect was also confirmed for $\mathrm{SrTiO}_{3}$ thin films deposited on various substrates. ${ }^{22}$ Only recently did Oh et al. ${ }^{23}$ report that bulk crystals of $\mathrm{Ca}_{3} \mathrm{Ti}_{2} \mathrm{O}_{7}$ and Sr-doped $\mathrm{Ca}_{3} \mathrm{Ti}_{2} \mathrm{O}_{7}$ with a $\mathrm{RP}$ phase show ferroelectricity. However, no ferroelectricity was reported for RP phases in a pseudo-binary SrO- $-\mathrm{TiO}_{2}$ system other than $\mathrm{SrTiO}_{3}$. Herein, we present the results of spontaneous superlattice structure formation in a Sr-Ti-O thin film deposited under a magnetic field that shows room-temperature ferroelectricity. We examined the origin of spontaneous superlattice formation and induced ferroelectricity for the Sr-excess thin film, which are the first instances of these phenomena in Sr-Ti-O thin films.

\section{EXPERIMENTAL PROCEDURE}

\section{Thin film deposition}

$\mathrm{Sr}-\mathrm{Ti}-\mathrm{O}$ thin films were deposited using PLD with a $\mathrm{KrF}$ excimer laser $\left(\lambda=248 \mathrm{~nm}, \quad 20 \mathrm{~ns}, \quad 7 \mathrm{~Hz}\right.$, fluence of $\sim 2 \mathrm{~J} \mathrm{~cm}^{-2}$ and spot size of $2.4 \times 10^{-2} \mathrm{~cm}^{2}$ ) at $700^{\circ} \mathrm{C}$ in oxygen at a pressure of $1.33 \times 10^{-2} \mathrm{~Pa}$. An electromagnet with a maximum magnetic field of $2 \mathrm{kG}$ was installed in the PLD chamber. ${ }^{24}$ Then, the effect of the application of the magnetic field during thin film deposition on crystallographic and electrical properties was examined. This PLD apparatus is designated as a dynamic aurora PLD. The schematic configuration showing an electromagnet, a target and a substrate, and a focused laser beam is shown in Figure 1a. A photograph of the electromagnet is presented in Figure 1b. For this study, a pair of thin films was deposited sequentially on both 0.5 at $\% \mathrm{Nb}$ doped $\mathrm{SrTiO}_{3}\left(\mathrm{Nb}-\mathrm{SrTiO}_{3}\right)$ (001) singlecrystal and $\mathrm{Si}$ substrates using identical deposition conditions. The film deposited on $\mathrm{Nb}_{-} \mathrm{SrTiO}_{3}$ (001) was used for crystallographic and electrical measurements. Films deposited on $\mathrm{Si}$ were used to measure thickness and composition. The film thickness was $300 \mathrm{~nm}$.

\section{Crystal structure characterization}

The in-plane and out-of-plane lattice parameters of epitaxial Sr-Ti-O thin film were measured using reciprocal space mapping measurements taken with highresolution X-ray diffractometry (ATX-G; Rigaku Corp., Tokyo, Japan). For the film deposited on the $\mathrm{Si}$ substrate, the composition and thickness were ascertained with an X-ray fluorescence spectrometer (Minipal; PANalytical B.V., Almelo, The Netherlands) equipped with the FP Multi software module. The film composition and thickness were confirmed using inductively coupled plasma atomic emission spectroscopy and a cross-sectional scanning electron microscopy (SEM) measurement, respectively. Cross-sectional scanning transmission electron microscopy (STEM) analysis was conducted using a STEM system with an accelerating voltage of $200 \mathrm{kV}$ (JEM-2100F; JEOL Ltd., Tokyo, Japan) equipped with an energy dispersive $\mathrm{x}$-ray spectroscopy (EDS) detector. High-resolution TEM analysis was conducted using TEM with accelerating voltage of $300 \mathrm{kV}$ (Titan 80-300; FEI Co., Hillsboro, OR, USA). The sample used for STEM and high-resolution transmission electron microscopy (HRTEM) observations was prepared using an ion milling system (EM-09100IS; JEOL Ltd.).

\section{Electrical property measurement}

The electrical properties of the films were measured after fabricating the top $\mathrm{Au}$ electrode $(100 \mu \mathrm{m} \phi)$ using dc sputtering. A Nb-SrTiO ${ }_{3}(001)$ single crystal was used as the bottom electrode. The $P-E$ curves and dielectric constant were measured using a ferroelectric tester (FC-E; Toyo Corp., Tokyo, Japan) and impedance analyzer (4194A; Agilent Technology Inc., Santa Clara, CA, USA).

\section{RESULTS AND DISCUSSION}

Figure 2a shows the X-ray diffraction (XRD) patterns of Sr-Ti-O thin films having a stoichiometric composition of $\mathrm{SrTiO}_{3}$ $(\mathrm{Sr} /(\mathrm{Sr}+\mathrm{Ti})=50.0$ at.\%) deposited with and without the application of a $2 \mathrm{kG}$ magnetic field during deposition. As this figure demonstrates, a rather broad perovskite (002) peak is observed at the lower angle of single-crystal $\mathrm{SrTiO}_{3}$ (002). The shift of the peak position of the $\mathrm{SrTiO}_{3}$ thin film to the low-angle side against the $\mathrm{SrTiO}_{3}$ single crystal means that the lattice of the $\mathrm{SrTiO}_{3}$ thin film deposited with and without the application of a magnetic field is expanded along the out-of-plane (c-axis) direction. The results further show that the application of a magnetic field during deposition enhanced the degree of lattice expansion, $\Delta c$, which is calculated as the difference of the out-of-plane lattice parameter of the thin films from that of bulk $\mathrm{SrTiO}_{3}$. The lattice expansion $(\Delta c)$ of $\mathrm{SrTiO}_{3}$ thin film was investigated extensively by Ohnishi et al. ${ }^{25}$ They found that the lattice expansion is brought about not by oxygen deficiency but by cation nonstoichiometry. They reported that the lattice expansion occurs for both Sr-excess and Ti-excess compositions. Because the films shown in Figure 2a have an overall stoichiometric composition and a rather broad peak, we infer that the lattice expansion results from randomly dispersed Sr-excess and Ti-excess regions in the film. Figure $2 \mathrm{~b}$ presents the XRD patterns of $\mathrm{Sr}-\mathrm{Ti}-\mathrm{O}$ thin films with Sr-excess compositions $(\mathrm{Sr} /(\mathrm{Sr}+\mathrm{Ti})=58.3$ at. $\%)$ deposited with and without the application of a $2 \mathrm{kG}$ magnetic field during deposition. The XRD pattern of the film deposited without the application of a magnetic field during deposition is fundamentally similar to that of the film with a stoichiometric composition shown in Figure 2a, except for the sharp diffraction peak. Surprisingly, the XRD pattern of the film deposited with the application of a magnetic field comprises a main peak (perovskite (002)) and six clearly defined satellite peaks with
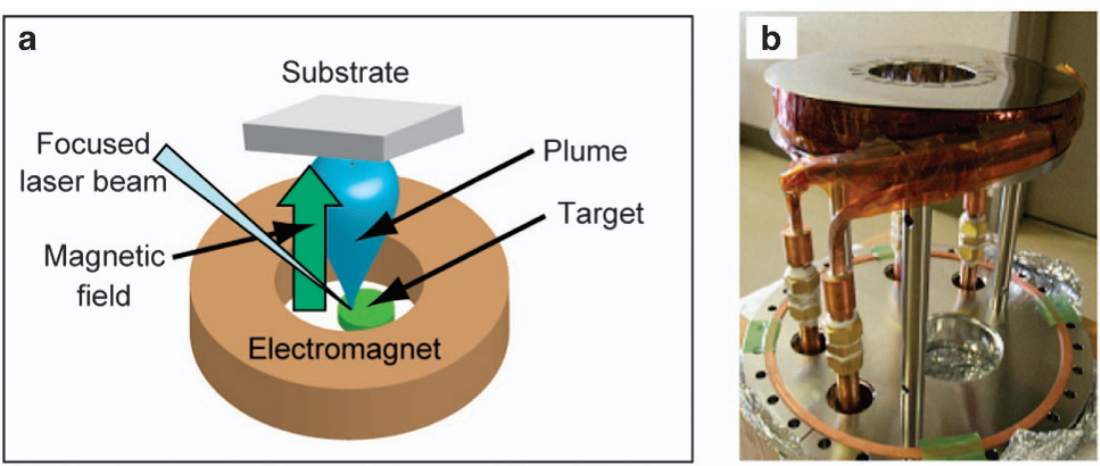

Figure 1 (a) Schematic configuration of an electromagnet, a target, a substrate and a focused laser beam. (b) Photograph of the electromagnet. 
a

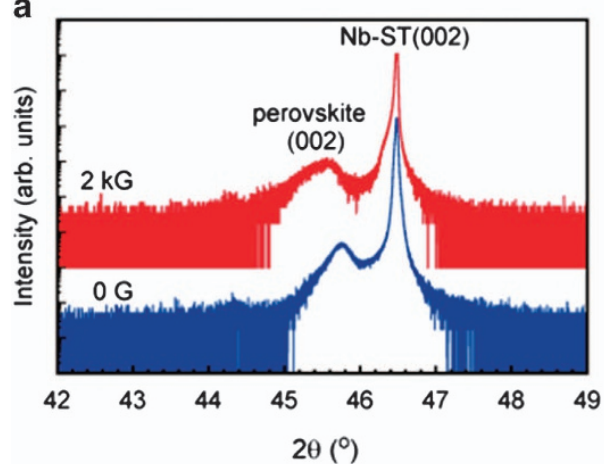

C

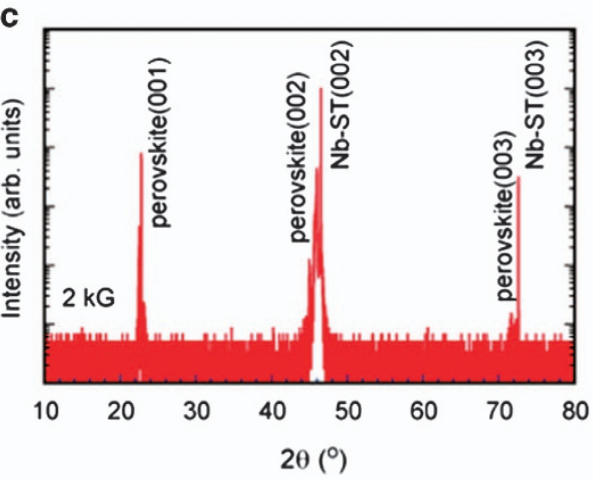

e

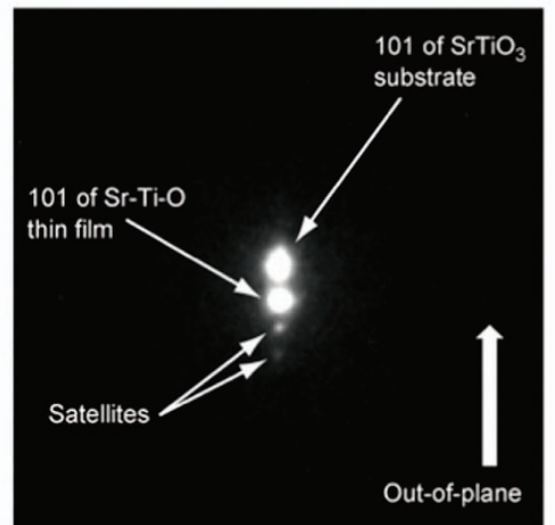

b

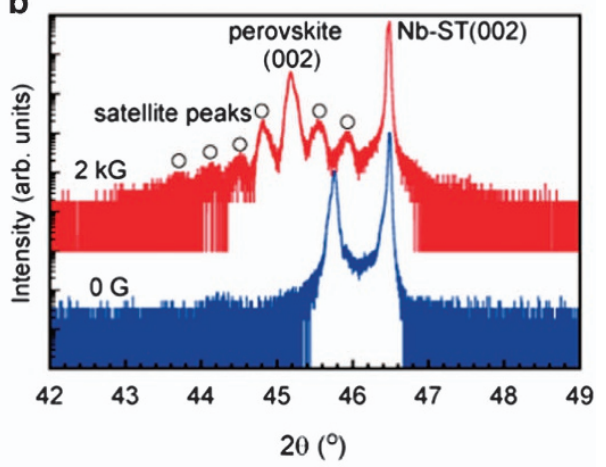

d

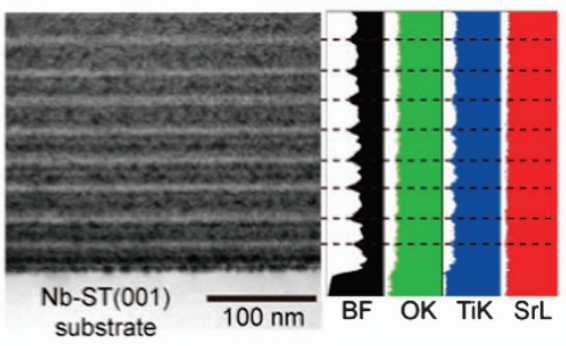

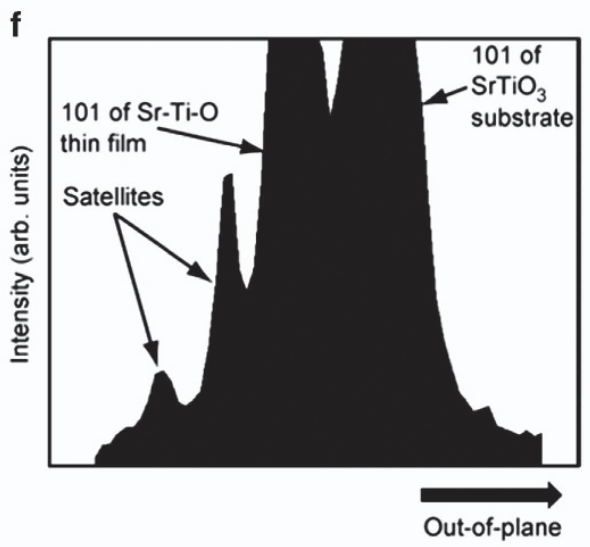

Figure 2 Crystal structures of $\mathrm{Sr}$-Ti-O thin films deposited on a Nb-doped $\mathrm{SrTiO}_{3}(001)$ single crystal. (a) XRD patterns for films deposited under $2 \mathrm{kG}$ and $0 \mathrm{G}$ magnetic fields for $\mathrm{Sr} /(\mathrm{Sr}+\mathrm{Ti})=50.0$ at. \% composition. (b) XRD patterns for films deposited under $2 \mathrm{kG}$ and $0 \mathrm{G}$ magnetic fields for $\mathrm{Sr} /(\mathrm{Sr}+\mathrm{Ti})=58.3$ at. $\%$ composition. The closed circle denotes the main peak. Open circles denote satellite peaks. (c) Wide range XRD pattern for the film with a spontaneously formed superlattice. (d) Cross-sectional STEM image of a film with intensities of bright field (BF) and EDS integrated line scans for O K, Ti K and Sr L for the film deposited under a $2 \mathrm{kG}$ magnetic field (shown in (b)). (e) Selected area electron diffraction patterns of cross sections of the Sr-Ti-O thin film deposited under a $2 \mathrm{kG}$ magnetic field (zone axis: (010)) (shown in (d)). (f) Histogram of the intensity of the electron diffraction along the out-of-plane direction (shown in (e)). Satellite spots prove the superlattice formation.

angular separation $\Delta \theta=0.187^{\circ}$, corresponding to a superlattice period, $\Lambda=25.6 \mathrm{~nm}$. The $\Lambda$ was calculated according to the following formula.

$$
\sin \theta_{m}=\sin \theta_{B} \pm m \lambda / 2 \Lambda
$$

Therein, $\theta_{\mathrm{B}}$ is the Bragg angle for the average film composition, $m$ is an integer (the order of reflection) and $\lambda$ is the X-ray wavelength $(0.15406 \mathrm{~nm}){ }^{26}$ Because the thickness of the film is $300 \mathrm{~nm}$, the satellite peaks are not caused by Laue oscillation, ${ }^{27}$ which suggests the spontaneous formation of a superlattice along the vertical direction of substrate. Because the composition of the film is A-site excess
$\left(\mathrm{Sr} /(\mathrm{Sr}+\mathrm{Ti})=58.3\right.$ at.\%, which corresponds to $\left.\mathrm{Sr}_{1.4} \mathrm{TiO}_{3.4}\right)$, the possibility of forming $\mathrm{Sr}_{3} \mathrm{Ti}_{2} \mathrm{O}_{7}$ exists. To consider this possibility, a wide range $\mathrm{XRD}$ scan was conducted (Figure 2c). If $\mathrm{Sr}_{3} \mathrm{Ti}_{2} \mathrm{O}_{7}$ is formed, the peaks of (008), (0012) and (0014) should be observed, respectively, near 35, 54 and $64^{\circ}$ in $2 \theta$. As shown in Figure 2c, no such peak was detected, which indicates that the diffraction pattern shown in Figure $2 \mathrm{~b}$ is derived not from $\mathrm{Sr}_{3} \mathrm{Ti}_{2} \mathrm{O}_{7}$ but from perovskite with a superlattice structure. To confirm the superlattice structure formation, crosssectional STEM observation and EDS-integrated line scans were conducted (Figure 2d). The left panel of Figure $2 \mathrm{~d}$ is a bright field 
a

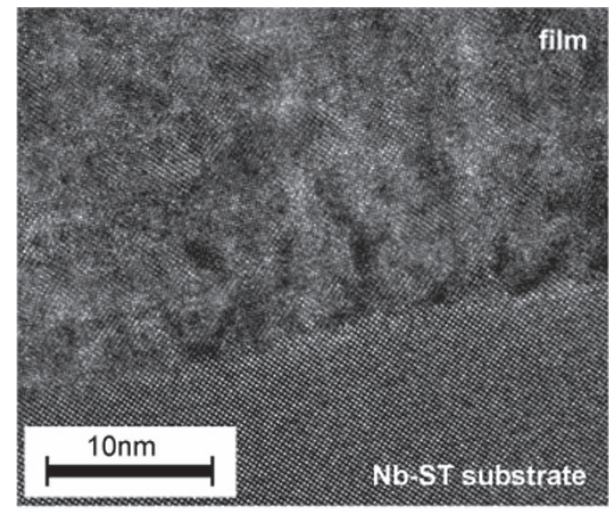

C

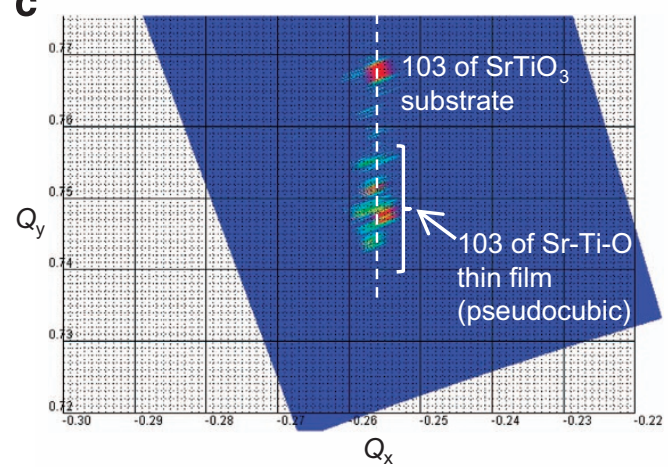

b

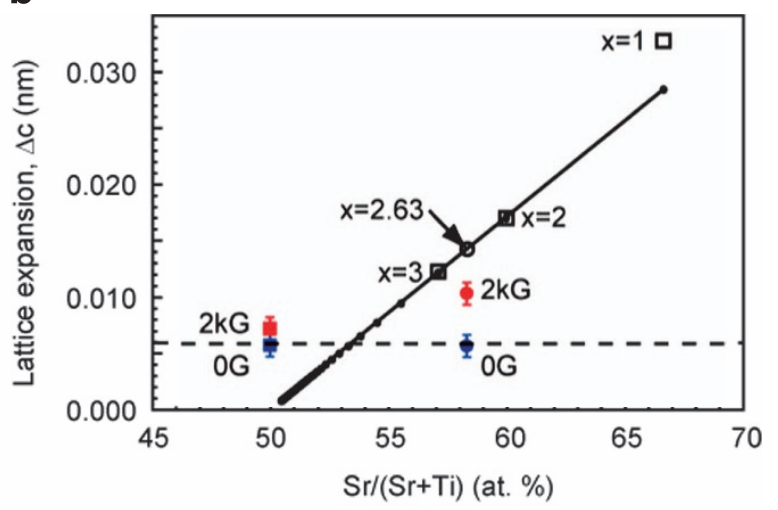

d

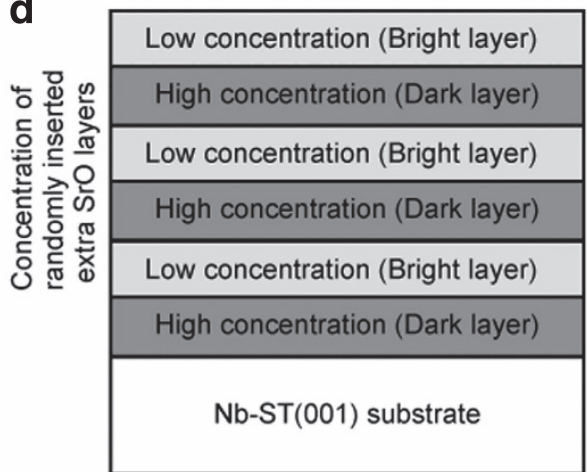

Figure 3 Crystal structures of $\mathrm{Sr}$-Ti-O thin films deposited onto a Nb-doped $\mathrm{SrTiO}_{3}(001)$ single crystal (part-2). (a) HRTEM image at the interface between the thin film and the $\mathrm{Nb}_{-} \mathrm{SrTiO}_{3}$ substrate for the sample presented in Figure $2 \mathrm{~d}$. (b) Change of lattice expansion ( $\Delta c$ ) with composition. The $\Delta c$ was calculated as the difference between the out-of-plane lattice parameter of the thin films from that of bulk $\mathrm{SrTiO}_{3}$. The changes of $\Delta c$ estimated by Ohnishi et al. ${ }^{25}$ for Ruddlesden-Popper $\left(\mathrm{SrTiO}_{3}\right)_{\mathrm{x}}-\mathrm{SrO}$ phases when treated as pseudocubic cells are also shown. (c) Reciprocal space map around $\mathrm{SrTiO}_{3}(103)$ for the film presented in Figure $2 b$. (d) Schematic drawing of spontaneously formed superlattice structure.

image of the film. This image shows that dark and bright layers are alternately ordered. The period of the ordered structure is approximately $30 \mathrm{~nm}$, which shows good agreement with the period determined using X-ray diffraction $(25.6 \mathrm{~nm})$. The right part of Figure $2 \mathrm{~d}$ shows the corresponding intensity of the bright field image and the result of the EDS line scans for O K, Ti K and Sr L lines. Although the $\mathrm{Sr} \mathrm{L}$ intensity is almost constant, the intensities of Ti $\mathrm{K}$ and $\mathrm{O} \mathrm{K}$ change periodically with the intensity of the bright field image, which shows that the superlattice structure was brought about by the composition modulation. Figure $2 \mathrm{e}$ shows the selected area electron diffraction pattern of the cross section of the Sr-Ti-O thin film deposited under a $2 \mathrm{kG}$ magnetic field (shown in Figure 2d). In this figure, diffraction spots of the $\mathrm{Nb}-\mathrm{SrTiO}_{3}$ substrate, the $\mathrm{Sr}-\mathrm{Ti}-\mathrm{O}$ thin film and satellites are observed along the out-of-plane direction. The electron diffraction intensity along the out-of-plane direction is shown as a histogram in Figure $2 \mathrm{f}$. The satellite peaks observed in this histogram also prove the superlattice formation from the point of cross-sectional STEM observation. As shown in Figure 2d, bright field images show that the film comprises alternately ordered dark and bright layers. To examine the detailed structure, the HRTEM image was observed at the interface between the thin film and $\mathrm{Nb}_{-}-\mathrm{SrTiO}_{3}$ single-crystal substrate (Figure 3a). Because the periodicity of the superlattice is approximately $30 \mathrm{~nm}$, the region shown as a film in Figure 3a depicts a part of the dark layer. The image shows that the film includes local and random structural changes, which might derive from randomly distributed RP type lattice displacements caused by the insertion of an extra $\mathrm{SrO}$ layer because the overall composition of the film is Sr-excess $(\mathrm{Sr} /(\mathrm{Sr}+\mathrm{Ti})=$ 58.3 at.\%). Such randomly distributed RP type defects are also reported by Ohnishi et al. ${ }^{25}$ for the epitaxial grown $\mathrm{SrTiO}_{3}$ thin film with a Srexcess composition. As described above, the Sr-Ti-O thin film diffraction peak is observed at a lower angle than that of the $\mathrm{SrTiO}_{3}$ single crystal, which suggests that the insertion of an extra SrO layer occurs at least along the out-of-plane (c-axis) direction. In addition, the HRTEM image shows that the SrO layer insertion occurs not only along the outof-plane (c-axis) direction but also along the in-plane (a-axis or b-axis) direction. To confirm this consideration, the relation between the lattice expansion along the out-of-plane (c-axis) direction $(\Delta c)$ with composition is shown in Figure 3b. For films deposited under a $0 \mathrm{G}$ magnetic field, the $\Delta c(=0.0058 \mathrm{~nm})$ was constant, irrespective of the composition. For the Sr-Ti-O thin film with a stoichiometric composition ( $\mathrm{Sr} /$ $(\mathrm{Sr}+\mathrm{Ti})=50.0$ at. $\%)$, the $\Delta c$ value was almost unchanged by the application of a magnetic field during deposition. However, for the film with a Sr-excess composition $(\mathrm{Sr} /(\mathrm{Sr}+\mathrm{Ti})=58.3$ at.\%), a considerable increase of $\Delta c(=0.0073 \mathrm{~nm})$ was observed, which suggests that the insertion of an additional SrO layer is enhanced by the application of the magnetic field during deposition. In this figure, the relation between $\Delta c$ and composition estimated by Ohnishi et al. ${ }^{25}$ for $\mathrm{RP}\left(\mathrm{SrTiO}_{3}\right)_{\mathrm{x}}-\mathrm{SrO}$ phases when treated as pseudocubic cells is also shown. In their estimation, $\Delta c$ was calculated under the assumption that the insertion of an extra SrO layer occurs only along the out-of-plane (c-axis) direction. 
a
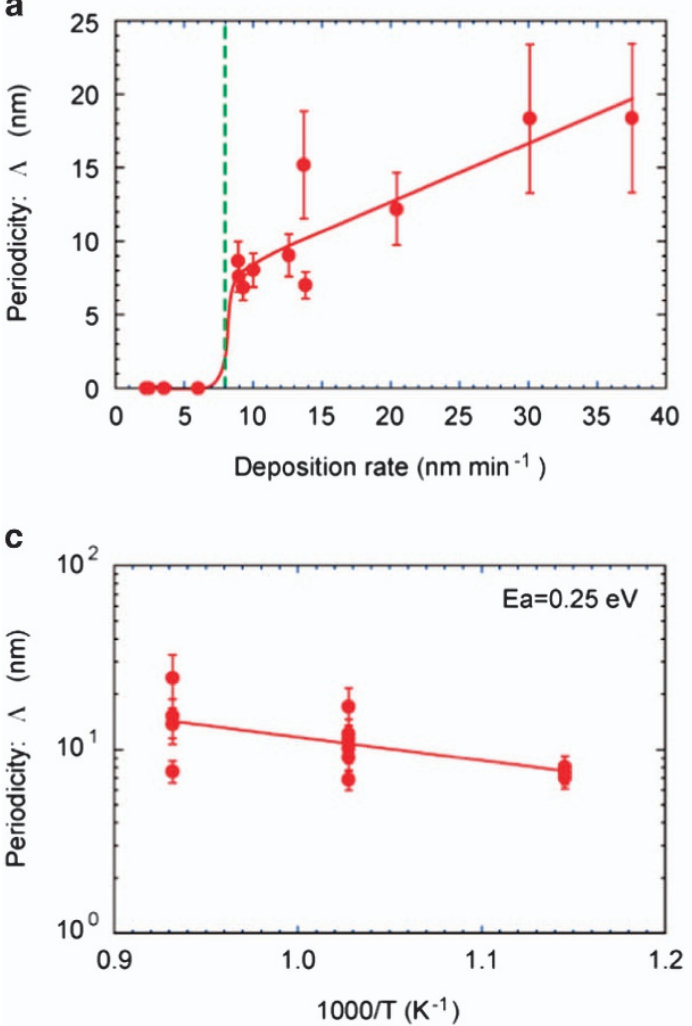

b

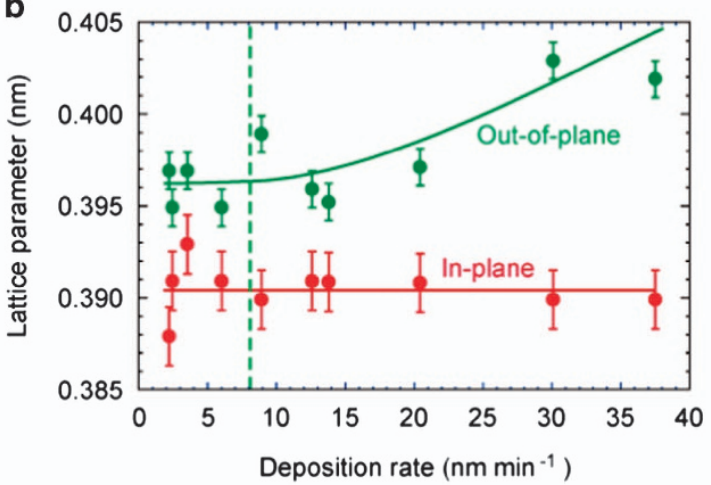

d

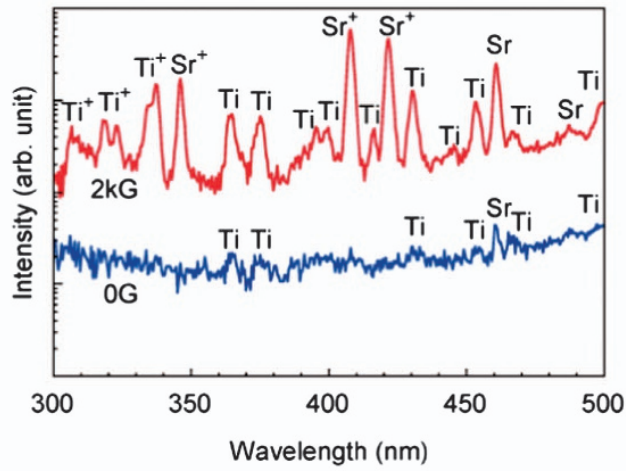

Figure 4 Characterization of spontaneous superlattice formation. (a) Change of periodicity with the deposition rate. (b) Change of the out-of-plane and in-plane lattice parameters with the deposition rate. (c) Arrhenius plot of periodicity. (d) Spectrogram of the plume with and without the application of a magnetic field during deposition.

For our thin film shown in Figures $2 \mathrm{~b}-\mathrm{f}$ and $3 \mathrm{a}$, the composition is $\mathrm{Sr} /(\mathrm{Sr}+\mathrm{Ti})=58.3$ at. $\%$. This composition corresponds to $x=2.63$ in $\left(\mathrm{SrTiO}_{3}\right)_{\mathrm{x}}$-SrO. If the insertion of an extra SrO layer occurs only along the out-of-plane (c-axis) direction, then $\Delta c$ is estimated as 0.0137 . However, the observed $\Delta c$ for the thin film deposited under a $2 \mathrm{kG}$ magnetic field is as low as 0.0073 . This supports the consideration that the insertion of additional SrO layer occurs not only along the out-of-plane direction but also along the in-plane direction. To ascertain whether such lattice expansion occurred along the in-plane direction or not, a reciprocal space map was measured around $\mathrm{SrTiO}_{3}$ (103) for the $\mathrm{Sr}-\mathrm{Ti}-\mathrm{O}$ thin film with a Sr-excess composition $(\mathrm{Sr} /(\mathrm{Sr}+\mathrm{Ti})=58.3$ at.\%) deposited under the magnetic field (Figure $3 \mathrm{c}$ ). In this figure, satellite peaks are also observed with the main peak along the $Q_{y}$ direction (out-of-plane direction), which agrees with the result obtained using XRD presented in Figure 2b. It is noteworthy that the $Q_{\mathrm{x}}$ value of the $\mathrm{Nb}-\mathrm{SrTiO}_{3}$ single-crystal coincides with that of the Sr-Ti-O thin film. Therefore, the Sr-Ti-O thin film is grown epitaxially on the $\mathrm{Nb}-\mathrm{SrTiO}_{3}$ (001) single-crystal substrate with coherent growth, showing that no lattice expansion occurs along the in-plane direction. Therefore, the film is under compressive stress. The film comprises alternately ordered dark and bright layers having different compositions. Therefore, we consider that both dark and bright layers consist of a perovskite phase, which consists of randomly inserted extra SrO layers. Moreover, the concentrations of extra SrO layers differ. We infer that the dark layer contains high concentrations of the extra SrO layer and that the bright layer contains low concentrations of the extra SrO layer. Therefore, the structure of the spontaneously formed superlattice can be shown schematically in Figure $3 \mathrm{~d}$.
To elucidate the mechanism of the spontaneously formed superlattice, we deposited many samples by changing the deposition rate, temperature and compositions. Figure $4 \mathrm{a}$ shows the change of periodicity of the superlattice with the deposition rate. This figure indicates that a threshold value exists for the deposition rate $\left(8 \mathrm{~nm} \mathrm{~min}{ }^{-1}\right)$ for the spontaneous formation of a superlattice. Beyond this value, periodicity increases with the deposition rate. A similar tendency by which the period increases with the deposition rate was also reported by Takahashi et al. ${ }^{14}$ for a $\mathrm{BaSnO}_{3}$ thin film. If the period is determined by thermal diffusion, then it should be inversely proportional to the growth rate. ${ }^{28,29}$ Figure $4 \mathrm{~b}$ shows the change of the in-plane and out-of-plane lattice parameters with the deposition rate. This figure shows the in-plane lattice parameter as constant within error, irrespective of the deposition rate. The in-plane lattice parameter coincides with that of bulk $\mathrm{SrTiO}_{3}$, as shown in Figure 3c. However, the out-of-plane lattice parameter increases with the deposition rate, which suggests that the out-of-plane strain changes with the deposition rate. Figure $4 \mathrm{c}$ presents an Arrhenius plot for the periodicity. As this figure shows, the activation energy for the spontaneous formation of the superlattice is $0.25 \mathrm{eV}$, which is much smaller than that reported for Ti diffusion in $\mathrm{SrTiO}_{3}(3.3 \mathrm{eV}) .{ }^{30}$ Reportedly, the application of a magnetic field during PLD enhances excitation and ionization in the plume. ${ }^{31-37}$ Actually, as shown in Figure $4 \mathrm{~d}$, weak emission peaks from neutral atoms ( $\mathrm{Sr}$ and $\mathrm{Ti}$ ) were observed in the plume when no magnetic field was applied. However, strong emission peaks associated with neutral and ionized atoms $\left(\mathrm{Sr}^{+}\right.$and $\left.\mathrm{Ti}^{+}\right)$were observed when a $2 \mathrm{kG}$ magnetic field was applied. Because the spectra were measured at the substrate position, 

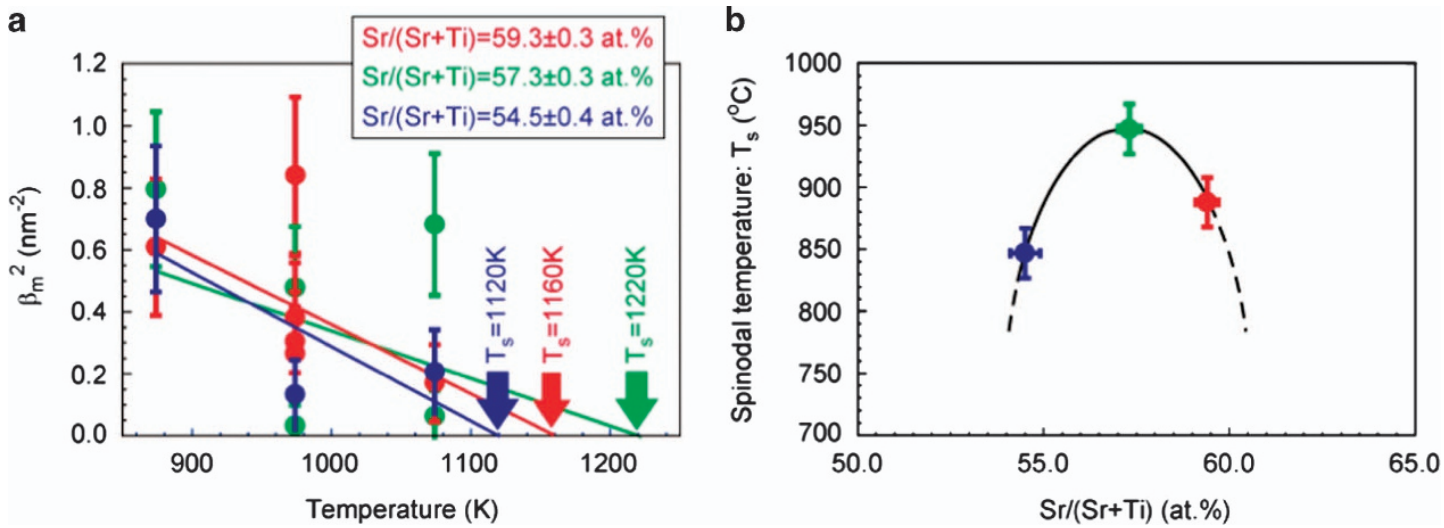

Figure 5 Determination of spinodal temperature $\left(T_{\mathrm{s}}\right)$. (a) Change of $\beta_{\mathrm{m}}{ }^{2}$ with temperature for $\mathrm{Sr} /(\mathrm{Sr}+\mathrm{Ti})=59.4,57.3$ and 54.5 at.\%. (b) Expected spinodal line for epitaxially grown $\mathrm{Sr}-\mathrm{Ti}-\mathrm{O}$ thin films.

ion-impingement (bombardment) occurs during deposition under the application of a magnetic field, which lowers the activation energy of diffusion according to the following equation: ${ }^{10}$

$$
D^{*}=D_{0} \exp \left(-\frac{Q-\alpha I}{\kappa T}\right)
$$

Therein, $D^{*}$ stands for the enhanced diffusion coefficient, $D_{0}$ denotes the pre-exponential factor, $Q$ signifies the activation energy without ion-impingement, $I$ represents the energy flux by the ion-impingement and $\alpha$ is a positive constant. Therefore, we infer that the low activation energy for the diffusion of $\mathrm{Ti}(0.25 \mathrm{eV})$ derives from this effect. In addition, lowering the activation energy brings about an increase of the diffusion coefficient that increases the period of

the superlattice $\Lambda$. The increase of $\Lambda$ with the deposition rate is explainable by this consideration. Because the diffusion enhances compositional modulation, the diffusion is up-hill diffusion, which is typical for the spinodal decomposition. According to Cahn and coworkers, ${ }^{38-41}$ the spinodal decomposition wavelength $\beta_{\mathrm{m}}$ can be given as shown below.

$$
\beta_{m}{ }^{2} \propto T_{s}-T
$$

Therein, $T_{\mathrm{s}}$ stands for the spinodal temperature.

Because $\beta_{\mathrm{m}}$ corresponds to the period of the superlattice, it is possible to ascertain $T_{\mathrm{s}}$ for several compositions by changing the deposition temperatures. Figure $5 \mathrm{a}$ shows the respective relations between $\beta_{\mathrm{m}}{ }^{2}$ and deposition temperature for $\mathrm{Sr} /(\mathrm{Sr}+\mathrm{Ti})$ compositions of $59.3 \pm 0.3$, $57.3 \pm 0.3$ and $54.5 \pm 0.4$ at $\%$. Although the data include a considerable range of error, the $T s$ for these compositions can be ascertained, respectively, as 1160,1220 and $1120 \mathrm{~K}$. Therefore, part of the spinodal curve for the Sr-Ti-O thin film was found, as shown in Figure 5b.

As described above, the Sr-Ti-O thin film with a spontaneously formed superlattice is under compressive stress. The symmetry is tetragonal. Therefore, for this work, the out-of-plane strain, $\varepsilon_{\mathrm{s}}$, is defined as the quotient of $\Delta c$ of the thin film described above and the lattice parameter of bulk $\mathrm{SrTiO}_{3}$ along the c-axis (which is equal to that along the a-axis), that is, $\varepsilon_{\mathrm{s}}=\Delta c$ (thin film)/c (bulk $\mathrm{SrTiO}_{3}$ ).

We prepared several samples exhibiting spontaneous superlattice formation by changing the deposition conditions. We measured the $P-E$ curves at room temperature. For these samples, the composition $\mathrm{Sr} /(\mathrm{Sr}+\mathrm{Ti})$ was $58.2 \pm 1.0$ at.\%. Figures $6 \mathrm{a}-\mathrm{f}$ show the variation of the $P-E$ curves with $\varepsilon_{s}$. The $\varepsilon_{\mathrm{s}}$ values for (a), (b), (c), (d), (e) and (f) are, respectively, $0.009,0.012,0.022,0.026,0.030$ and 0.036. Sample (a) shows the paraelectric property. Sample (b) is also paraelectric. However, a slight tendency of ferroelectricity is detectable. Samples (c), (d), (e) and (f) show nonlinearity in the P-E curves, suggesting slight ferroelectricity with slight remanent polarization of less than $5 \mu \mathrm{C} \mathrm{cm}^{-2}$. As demonstrated in Figures $6 \mathrm{c}$ and $\mathrm{d}$, the remanent polarization increases slightly with $\varepsilon_{s}$. However, the remanent polarization does not increase with $\varepsilon_{\mathrm{s}}$ for (e) or (f). This result suggests that the ferroelectricity in the spontaneously formed superlattice is not decided simply by the strain alone; thus, other factors such as the periodicity of the superlattice should be considered. To clarify, further investigation is needed. In Figure $6 \mathrm{~d}$, a $P$-E curve measured for a thin film with a stoichiometric composition deposited under a magnetic field is also shown for comparison, exhibiting no ferroelectricity. These results indicate that induced ferroelectricity is observed for a Sr-Ti-O thin film deposited under a magnetic field with a Sr-excess composition. Films deposited without the application of a magnetic field and which did not exhibit superlattice formation showed no ferroelectricity. To confirm the induced ferroelectricity, the temperature dependence of the dielectric constant was measured for samples (e) and (f), as shown in Figures $6 \mathrm{e}^{\prime}$ and $\mathrm{f}^{\prime}$. These samples, respectively, showed maximum $\varepsilon_{\mathrm{r}}$ values $\left(T_{\mathrm{c}}\right)$ at 340 and $420^{\circ} \mathrm{C}$. These data also show that $T_{\mathrm{c}}$ increases with the $\varepsilon_{\mathrm{s}}$ value. Unfortunately, the measurement of temperature dependence for other samples failed because of leakage and/or difficulties with the top electrode during the experiment. As described above, Haeni et al. ${ }^{21}$ reported roomtemperature ferroelectricity for strained $\mathrm{SrTiO}_{3}$ and predicted the change of transition temperature with in-plane strain. They calculated the in-plane strain, $\varepsilon_{s}$, according to the in-plane lattice mismatch between the $\mathrm{SrTiO}_{3}$ thin film and the substrate, that is, $\varepsilon_{\mathrm{s}}=\mathrm{a}\left(\mathrm{SrTiO}_{3}\right.$ thin film) - a(substrate). Because the ferroelectricity induced by inplane strain fundamentally corresponds to that because of out-ofplane strain, one can show the relation between temperature and outof-plane strain, $\varepsilon_{\mathrm{s}}$, for samples (a)-(f), as an extension of the work by Haeni et al. in Figure 6g. It is noteworthy that the sign is reversed by the change of definition. Compressive strain is negative for the inplane strain, but it is positive for the out-of-plane strain. As shown in this figure, the experimentally obtained results are clearly explainable: sample (a) is paraelectric; sample (b) is marginally ferroelectric; samples (c), (d), (e) and (f) are ferroelectric. Furthermore, the temperature dependence for samples (e) and ( $\mathrm{f}$ ) is explainable because the observed $T_{c}$ values are close to the range of the ferroelectric to paraelectric transition. This fact suggests that the room-temperature 

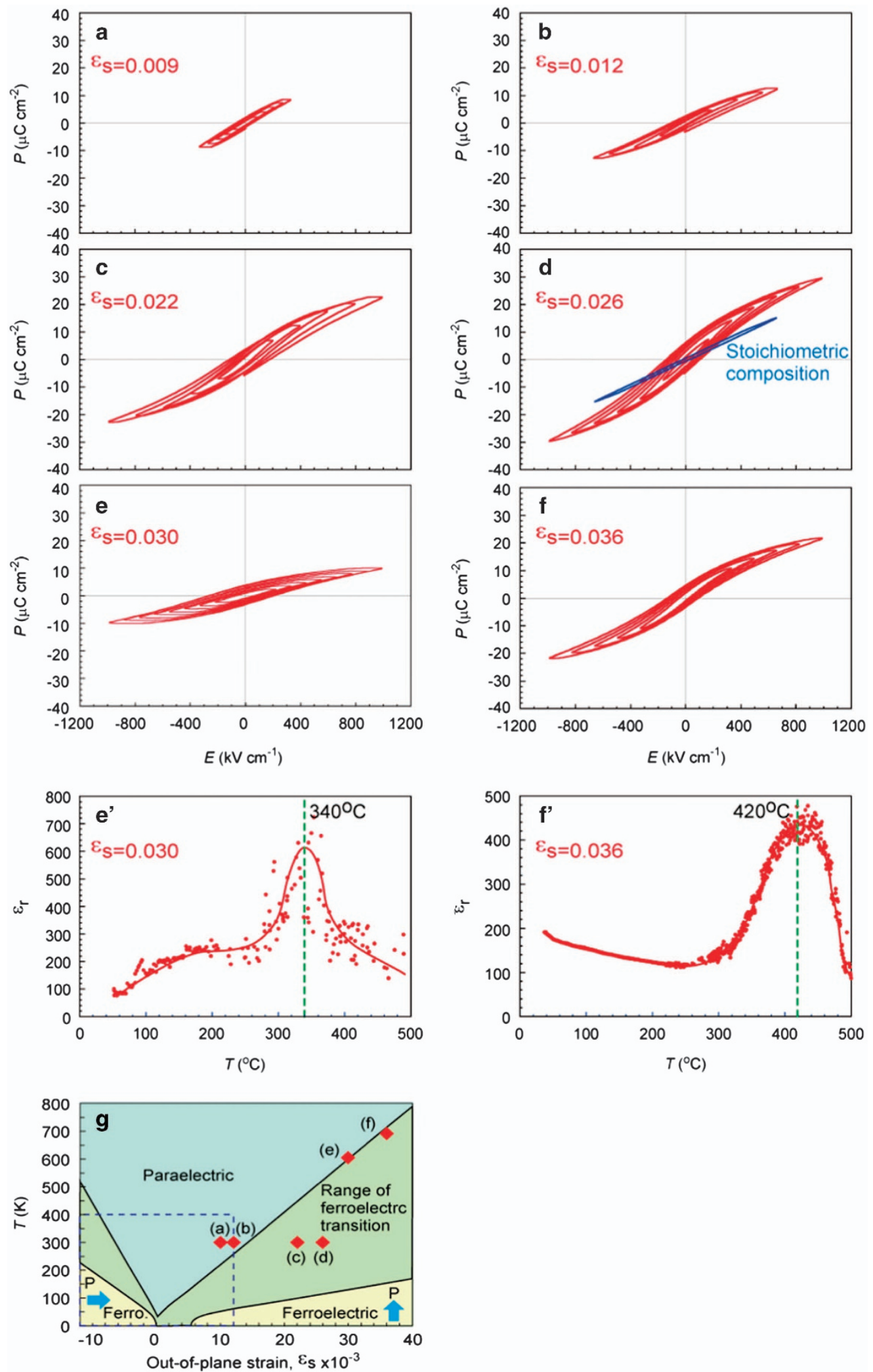

Figure 6 Ferroelectric properties of the Sr-Ti-O thin films deposited under a $2 \mathrm{kG}$ magnetic field as a function of the out-of-plane strain, $\varepsilon_{\mathrm{S}}$. (a-f) $P$ - $E$ curves, respectively, of films with $\varepsilon_{\mathrm{s}}$ values of $0.009,0.012,0.022,0.026,0.030$ and 0.036 . In (d), data for stoichiometric composition are also shown. (é $\mathbf{e}^{\prime}$ and $\mathbf{f}^{\prime}$ ) show the temperature dependence of the dielectric constant for $\varepsilon_{\mathrm{s}}$ values of 0.030 and 0.036 , respectively. (g) The extension of the expected shift in $T_{\mathrm{c}}$ of $\mathrm{SrTiO}_{3}(001)$ with biaxial strain reported by Haeni et al. ${ }^{21}$ (box in dashed line) and data are shown in (a-f). 
ferroelectricity observed for our films is clearly explainable based on thermodynamic phenomenology.

The main point of this work is the finding that a spontaneous superlattice formation occurs in a Sr-Ti-O system (A-site excess $\mathrm{SrTiO}_{3}$ ) caused by spinodal decomposition during thin film deposition. The spinodal decomposition brings about an alternative layer structure. Because the c-axis of the film was elongated and because no coexistence of secondary phases was detected, spontaneously formed alternative layers are believed to comprise $\mathrm{SrTiO}_{3}$ with randomly inserted extra SrO layers (RP defects). In addition, the degree of insertion differs for the two layers formed as a result of spinodal decomposition. In addition, it is important that the films show room-temperature ferroelectricity. In the literature, strain-induced ferroelectricity of $\mathrm{SrTiO}_{3}$ thin films was reported mainly along the inplane direction. ${ }^{21,22,42}$ Very few reports describe phenomena along the out-of-plane direction. ${ }^{43-45}$ The out-of-plane ferroelectricity reported by Warusawithana et al. ${ }^{43}$ is limited in terms of thickness (less than $10 \mathrm{ML}$ ) and that reported by Kim et al. ${ }^{44,45}$ originates from vacancies. Therefore, this report is the first to describe out-of-plane ferroelectricity caused by strain in $\mathrm{SrTiO}_{3}$ thin film with significant thickness. Currently, PLD is widely used worldwide. Therefore, the fact that the application of a magnetic field during deposition brings about spontaneous superlattice formation and enhanced properties is expected to open new avenues for materials science investigations.

\section{CONFLICT OF INTEREST}

The authors declare no conflict of interest.

\section{ACKNOWLEDGEMENTS}

A part of this study was supported by the Tohoku University microstructural characterization platform as a program of the 'Nanotechnology Platform' of the Ministry of Education, Culture, Sports, Science and Technology (MEXT) of Japan. This work was performed under the Inter-university Cooperative Research Program of the Institute for Materials Research, Tohoku University (Proposal No. 15K0049). We also gratefully acknowledge A. Akama of Tohoku University for fabricating a cross-sectional sample for TEM observation. We thank T. Kubota, T. Sakakibara, D. Suzuki, H. Ishii and K. Yamashita for experiments and fruitful discussions. This work was supported by a Grant-inAid for Scientific Research from the Ministry of Education, Culture, Sports, Science and Technology (No. 15H04123). This work was conducted under the Cooperative Research Project Program of the Research Institute of Electronics, Shizuoka University (Grant Nos. H27-10 and H27-11). Part of this work was supported by the Concert-Japan Project (FF-Photon) from the Japan Science and Technology Agency. We gratefully acknowledge Toyo Corporation for fruitful discussions on ferroelectric measurements.

1 Petroff, P. M., Cho, A. Y., Reinhart, F. K., Gossard, A. C. \& Wiegmann, W. Alloy clustering in Ga1-xAlxAs compound semiconductors grown by molecular beam epitaxy. Phys. Rev. Lett. 48, 170-173 (1982).

2 Pakula, K., Borysiuk, J., Bozek, R. \& Baranowski, J. M. Long-range order spontaneous superlattice in AIGaN epilayers. J. Cryst. Growth 296, 191-196 (2006).

3 Wu, Z. H., Kawai, Y., Fang, Y.-Y., Chen, C. Q., Kondo, H., Hori, M., Honda, Y., Yamaguchi, M. \& Amano, H. Spontaneous formation of highly regular superlattice structure in InGaN epilayers grown by molecular beam epitaxy. Appl. Phys. Lett. 98, 141905 (2011).

4 Ahrenkiel, S. P., Xin, S. H., Reimer, P. M., Berry, J. J., Luo, H., Short, S., Bode, M., Al-Jassim, M., Buschert, J. R. \& Furdyna, J. K. Self-organized formation of compositionally modulated ZnSe1-xTex superlattices. Phys. Rev. Lett. 75, 1586-1589 (1995).

5 Venezuela, P., Tersoff, J., Floro, J. A., Chason, E., Follstaedt, D. M., Liu, F. \& Lagally, M. G. Self-organized growth of alloy superlattices. Nature 397, 678-681 (1999).

6 Chin, A., Lin, B. C., Gu, G. L. \& Hsieh, K. Y. Spontaneously formed long-range Al-rich

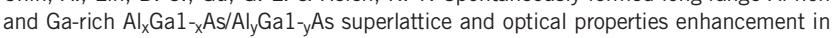
(111)A AlGaAs. Appl. Phys. Lett. 67, 3617-3619 (1995).
7 Ebling, D. G., Kirste, L., Benz, K. W., Teofilov, N., Thonke, K. \& Sauer, R. Optical properties and ordering of $\mathrm{Al}_{x} \mathrm{Ga}{ }_{-} \mathrm{N}$ MNE-layers. J. Cryst. Growth 227-228, 453-457 (2001).

8 Daruka, I. \& Tersoff, J. Self-assembled superlattice by spinodal decomposition during growth. Phys. Rev. Lett. 95, 076102 (2005).

9 He, J. H., Carosella, C. A., Hubler, G. K., Qadri, S. B. \& Sprague, J. A. Bombardmentinduced tunable superlattices in the growth of Au-Ni films. Phys. Rev. Lett. 96, 056105 (2006).

10 Chen, C. Q., Pei, Y. T., Shaha, K. P. \& Hosson, J. Th. M. De. Tunable self-organization of nanocomposite multilayers. Appl. Phys. Lett. 96, 073103 (2010).

$11 \mathrm{Liu}$, J., Wu, X., Lennard, W. N. \& Landheer, D. Surface-directed spinodal decomposition in hafnium silicate thin films. Phys. Rev. B 80, 041403(R) (2009).

12 Yang, H., Wang, H., Maiorov, B., Lee, J., Talbayev, D., Hinton, M. J., Feldmann, D. M., MacManus-Driscoll, J. L., Taylor, A. J., Civale, L., Lemberger, T. R. \& Jia, Q. X. Self-assembled multilayers and enhanced superconductivity in $\left(\mathrm{YBa}_{2} \mathrm{Cu}_{3} \mathrm{O}_{7}-\mathrm{x}\right)_{0.5}$ : $\left(\mathrm{BaZrO}_{3}\right)_{5}$ nanocomposite films. J. Appl. Phys. 106, 093914 (2009).

13 MacManus-Driscoll, J. L. Self-assembled Heteroepitaxial oxide nanocomposite thin film structures: Designing interface-induced functionality in electric materials. Adv. Func. Mater 20, 2035-2045 (2010).

14 Takahashi, R., Valset, K., Folven, E., Eberg, E., Grepstad, J. K. \& Tybell, T. Long-range spontaneous structural ordering in barium stannate thin films. Appl. Phys. Lett. 97, 081906 (2010).

15 Ruddlesden, S. N. \& Popper, P. The compound $\mathrm{Sr}_{3} \mathrm{Ti}_{2} \mathrm{O}_{7}$ and its structure. Acta Crystallogr 11, 54-55 (1958).

16 Barrett, J. H. Dielectric constant in perovskite type crystals. Phys. Rev 86, 118-120 (1952).

17 Fleury, P. A., Scott, J. F. \& Worlock, J. M. Soft phonon modes and the $110^{\circ} \mathrm{K}$ phase transition in $\mathrm{SrTiO}_{3}$. Phys. Rev. Lett. 21, 16-19 (1968).

18 Bednorz, J. G. \& Müller, K. A. Sr1-x $\mathrm{CaxTiO}_{3}$ : An XY quantum ferroelectric with transition to randomness. Phys. Rev. Lett. 52, 2289-2292 (1984).

19 Itoh, M., Wang, R., Inaguma, Y., Yamaguchi, T., Shan, Y.-J. \& Nakamura, T. Ferroelectricity induced by oxygen isotope exchange in strontium titanate perovskite. Phys. Rev. Lett. 82, 3540-3543 (1999).

20 Uwe, H. \& Sakudo, T. Stress-induced ferroelectricity and soft phonon modes in $\mathrm{SrTiO}_{3}$. Phys. Rev. B 13, 271-286 (1976).

21 Haeni, J. H., Irvin, P., Chang, W., Uecker, R., Reiche, P., Li, Y. L., Choudhury, S., Tian, W., Hawley, M. E., Craigo, B., Tagantsev, A. K., Pan, X. Q., Streiffer, S. K., Chen, L. Q., Kirchoefer, S. W., Levy, J. \& Schlom, D. G. Room-temperature ferroelectricity in strained $\mathrm{SrTiO}_{3}$. Nature 430, 758-761 (2004).

22 Wördenweber, R., Hollmann, E., Kutzner, R. \& Schubert, J. Induced ferroelectricity in strained epitaxial $\mathrm{SrTiO}_{3}$ films on various substrates. J. Appl. Phys. 102, 044119 (2007).

23 Oh, Y. S., Luo, X., Huang, F. T., Wang, Y. \& Cheong, S. W. Experimental demonstration of hybrid improper ferroelectricity and the presence of abundant charged walls in $(\mathrm{Ca}, \mathrm{Sr})_{3} \mathrm{Ti}_{2} \mathrm{O}_{7}$ crystals. Nature Mater 14, 407-413 (2015).

24 Wakiya, N., Muraoka, K., Kadowaki, T., Kiguchi, T., Mizutani, N., Suzuki, H. \& Shinozaki, K. Preparation of ferromagnetic zinc-ferrite thin film by pulsed laser deposition in the magnetic field. J. Mag. Mag. Mater 310, 2546-2548 (2007).

25 Ohnishi, T., Shibuya, K., Yamamoto, T. \& Lippmaa, M. Defects and transport in complex oxide thin films. J. Appl. Phys. 103, 103703 (2008).

26 Schuller, I. K. New class of layered materials. Phys. Rev. Lett. 44, 1597-1600 (1980).

$27 \mathrm{Mau}$, M., Schulte, B., Häussler, P. \& Adrian, H. $\mathrm{YBa}_{2} \mathrm{Cu}_{3} \mathrm{O}_{7-\delta}$ thin films on sapphire with buffer layers of CeO2. Physica B: Condensed Matter 194-196, 2285-2286 (1994).

28 Barabasi, A. L. Self-organized superlattice formation in II-IV and III-V semiconductors. Appl. Phys. Lett. 70, 764-766 (1997).

29 Zheng, H., Straub, F., Zhan, Q., Yang, P. L., Hsieh, W. K., Zavaliche, F., Chu, Y. H., Dahmen, U. \& Ramesh, R. Self-assembled growth of $\mathrm{BiFeO}_{3}-\mathrm{CoFe}_{2} \mathrm{O}_{4}$ nanostructures. Adv. Mater. 18, 2747-2752 (2006).

30 Gömann, K., Borchardt, K. G., Gunhold, A., Maus-Friedrichs, W. \& Baumann, H. Ti diffusion in La-doped $\mathrm{SrTiO}_{3}$ single crystals. Phys. Chem. Chem. Phys. 6, 3639-3644 (2004).

31 Dirnberger, L., Dyer, P. E., Farrar, S. R. \& Key, P. H. Observation of magnetic-fieldenhanced excitation and ionization in the plume of KrF-laser-ablated magnesium. Appl. Phys. A 59, 311-316 (1994).

32 Lash, J. S., Gilgenbach, R. M. \& Ching, C. H. Laser-ablation-assisted-plasma discharges of aluminum in a transverse-magnetic field. Appl. Phys. Lett. 65, 531-533 (1994).

33 Kokai, F., Koga, Y. \& Heimann, R. B. Magnetic field enhanced growth of carbon cluster ions in the laser ablation plume graphite. Appl. Surf. Sci. 96-98, 261-266 (1996).

34 Takeuchi, M. \& Kobayashi, T. Manipulation of laser plume by magnetic field application. Jpn. J. Appl. Phys. 38, 3642-3645 (1999).

35 Kokai, F., Yamamoto, K., Koga, Y., Fujiwara, S. \& Heimann, R. B. Characterization of ablation plumes and carbon nitride films produced by reactive pulsed laser deposition in the presence of a magnetic field. Appl. Phys. A 66, 403-406 (1998).

36 Kobayashi, T., Akiyoshi, H. \& Tachiki, M. Development of prominent PLD (Aurora method) suitable for high-temperature film growth. Appl. Surf. Sci. 197-198, 294-303 (2002).

37 García, T., de Posada, E., Villagrán, M., Sánchez LI, J. L., Bartolo-Pérez, P. \& Peña, J. L. Effects of an external magnetic field in pulsed laser deposition. Appl. Surf. Sci. 255, 2200-2204 (2008).

38 Cahn, J. W. \& Hilliard, J. E. Free energy of a nonuniform system. I. Interfacial free energy. J. Chem. Phys. 28, 258-267 (1958).

39 Cahn, J. W. On spinodal decomposition. Acta Mat. 9, 795-801 (1961). 
40 Cahn, J. W. On spinodal decomposition in cubic crystals. Acta Mat. 10, 179-183 (1962).

41 Cahn, J. W. Phase separation by spinodal decomposition in isotropic systems. J. Chem. Phys. 42, 93-99 (1965).

42 Biegalski, M. D., Jia, Y., Schlom, D. G., Trolier-McKinstry, S., Streiffer, S. K., Sherman, V., Uecker, R. \& Reiche, P. Relaxor ferroelectricity in strained epitaxial $\mathrm{SrTiO}_{3}$ thin films on $\mathrm{DyScO}_{3}$ substrates. Appl. Phys. Lett. 88, 192907 (2006).

43 Warusawithana, M. P., Cen, C., Sleasman, C. R., Woicik, J. C., Li, Y., Kourkoutis, L. F., Klug, J. A., Li, H., Ryan, P., Wang, L. P., Bedzyk, M., Muller, D. A., Chen, L. Q., Levy, J. \& Schlom, D. G. A ferroelectric oxide made directly on silicon. Science 324, 367 (2009).

44 Kim, Y. S., Kim, D. J., Noh, T. W., Choi, J. S., Park, B. H. \& Yoon, J. G. Observation of room-temperature ferroelectricity in tetragonal strontium titanate thin films on $\mathrm{SrTiO}_{3}$ (001) substrates. Appl. Phys. Lett. 91 042908 (2007).
45 Kim, Y. S., Kim, J., Moon, S. J., Choi, W. S., Chang, Y. J., Yoon, J.-G., Yu, J., Chung, J.-S. \& Noh, T. W. Localized electronic states induced by defects and possible origin of ferroelectricity in strontium titanate thin film. Appl. Phys. Lett. 94, 202906 (2009).

(c) (i) This work is licensed under a Creative Commons Attribution 4.0 International License. The images or other third party material in this article are included in the article's Creative Commons license, unless indicated otherwise in the credit line; if the material is not included under the Creative Commons license, users will need to obtain permission from the license holder to reproduce the material. To view a copy of this license, visit http:// creativecommons.org/licenses/by/4.0/ 